\title{
SCIENCES NATURELLES, BIOLOGIE, MÉDECINE
}

Buffon : 1788-1988. Paris, Imprimerie nationale, 1988. $21 \times 27,296$ p., ill. en noir et en coul.

L'ouvrage, célébrant le bicentenaire de la mort de Buffon (1707-1788), est destiné au large public et bénéficie de signatures scientifiques. A ce titre, il comporte des textes d'inégale valeur historiographique. Dans un genre panégyrique, on apprend que Buffon annonce "quelque peu ", par sa théorie des molécules organiques, "ce que dira bien plus tard Jacques Monod"; qu'il fut "sincèrement convaincu de la réalité de l'évolution "; qu' "il préfigure ce que dira bien plus tard François Jacob qui proclame que l'homme n'est programmé que pour apprendre. Rien n'aura échappé à sa pensée " (J. Dorst)! Au-delà des quelques textes de circonstances, pesamment anachroniques ou hagiographiques, Bernard Rignault et Serge Benoît ont choisi de présenter l'une des facettes de Buffon parmi les moins connues du grand public. Sur les terres de Buffon, il avait créé le "plus considérable établissement" sidérurgique de Bourgogne, devenu, grâce à l'action de l'Association de sauvegarde des Forges de Buffon, un monument précieux et l'un des plus beaux exemples de restauration de l'archéologie industrielle.

Philosophe spéculatif, Buffon était tout autant entrepreneur et homme d'affaires, ce que l'on constate sans difficulté à la lecture des épisodes de sa vie bourguignonne et des plans de rénovation du Jardin du Roi, rappelés ici par Yves Laissus. Il est moins connu, mais tout aussi instructif, de noter que cette perspective d'entrepreneur, de domination rationnelle de la nature brute " hideuse et mourante » selon ses mots, permet à Buffon d'offrir une image dynamique de la nature humaine, toute de projets, de risques calculés. La " valeur " de l'Homme, telle qu'il la réfléchit sur ce mode productiviste, n'est ni donnée d'avance, ni inaliénable. L'essence abstraite de la perfectibilité d'espèce implique, pour se réaliser, le passage à l'acte qui la produit et la reproduit sans cesse; elle suppose le travail qui la matérialise, par la mise en valeur du sol cultivé ou la maitrise technique des forces naturelles. Cette projection active de la pensée humaine dans l'avenir d'un monde ordonné et comme pacifié est présente en bien des pages classiques de Buffon. Les plus explicites d'entre elles, et notamment les « Vues " sur la nature et la "Septième époque de la Nature », sont ici reproduites. On y remarque à quel point la conception buffonienne de la place de l'homme dans la nature est tissée d'éléments biographiques. Buffon montre que l'homme, en 
intervenant dans le monde par sa prise technique, convertit un réel hostile en totalité de sens, finalisée pour lui. La « nature cuitivée ", " brillante et pompeusement parée " devient le miroir où se réfléchit la grandeur de l'homme.

Dans un autre registre, l'épistémologie de Buffon est ici doublement représentée, par la réédition du Mémoire d'ouverture à l'Histoire naturelle, intitulé " De la manière d'étudier et de traiter l'histoire naturelle » (1749) et par la reprise de "l'Introduction" de Jean Piveteau, qui ouvrait les Euvres philosophiques de Buffon publiées en 1954. Ce texte de synthèse n'a malheureusement pas été actualisé pour tenir compte du progrès des études buffoniennes. Il y a toutefois une bibliographie complémentaire (en langue française seulement).

Mais, et c'est là l'essentiel pour sa destination, l'ouvrage est magnifiquement illustré. De nombreuses planches d'animaux dessinées par de Sève sont reproduites, également des portraits et bustes de Buffon présentés par Paul-Marie Grinevald, des vues de Montbard et des Forges de Buffon. La qualité de l'iconographie et de la conception graphique suffirait à justifier l'acquisition de ce livre. Pour le lecteur non spécialisé les textes fournissent un bon complément pour leur intelligibilité. Le lecteur pourra encore se faire une juste idée du style de Buffon sur quelques-unes de ses pages les plus philosophiques et comparer utilement le "discours de réception à l'Académie française » et l'application de ses préceptes.

Claude BlanckaerT.

Julien-Joseph Virey, naturaliste et anthropologue. Sous la dir. de Claude BÉNICHOU et de Claude Blanckaert. Paris, Vrin, 1988. $15 \times 20,7,289$ p., bibliogr., index ( Sciences en situation, histoire, épistémologie, vulgarisation $»)$.

Parmi les naturalistes français, Julien-Joseph Virey n'occupe assurément qu'une place modeste. Ne jouissant ni de la gloire littéraire de Buffon, ni du prestige scientifique de Lamarck ou de Cuvier, il ne figure plus guère dans les histoires des sciences qu'à titre de tenant d'une anthropologie dépassée. Aussi, consacrer à Virey une journée d'étude, qui s'est tenue le 29 novembre 1986 à Paris XII-Créteil, et en publier les Actes relevait de la gageure.

En fait, dès l'avant-propos de Claude Bénichou et Claude Blanckaert, on perçoit comment, pour une histoire des sciences désireuse de sortir du paradigme bachelardien et de ne pas se limiter à retracer les «formations progressives de la vérité ", un personnage de second plan comme Virey peut représenter un thème d'étude au même titre que tel ou tel grand auteur. Au lieu de prendre pour une évidence le jugement de la postérité distribuant les différents degrés de la gloire, les auteurs entendent penser historiquement ce qui donne à un auteur tel ou tel statut à l'intérieur de sa discipline.

Jacques Léonard trace ensuite en quelques pages un portrait de l'homme et du naturaliste. Né en 1775, Julien-Joseph Virey, après ses études au collège de Langres, entre en apprentissage chez un oncle apothicaire. Pharmacien militaire, 
contemporain des guerres de la Révolution et de l'Empire, il passe près de vingt ans de sa vie dans le service de santé des armées. Influencé par Parmentier, qui l'a aidé dans ses débuts, il écrit plusieurs textes sur l'hygiène alimentaire et sur les médicaments. En 1814, il passe son doctorat en médecine mais se présente surtout comme un hygiéniste. Cette pratique professionnelle n'est cependant qu'un des versants de sa formation et de son activité, l'autre étant la fréquentation assidue des bibliothèques qui lui permet d' " accumuler une immense érudition " (p. 21). Dans cette érudition, il puise les matériaux d'une abondante production et en particulier d'un grand nombre d'articles de dictionnaires. Au moment de sa mort en 1846, il passe aux yeux de beaucoup de ses confrères pour un vulgarisateur superficiel. Certains de ses contemporains lui reprochent par surcroît une attitude trop effacée comme député de la Haute-Marne sous la Monarchie de Juillet. C'est ce qu'attestent les quelques textes de l'époque reproduits en annexe (p. 23-30).

Pharmacien, médecin, érudit, polygraphe, député, il reste en même temps un naturaliste et c'est sur cet aspect que porte l'essentiel des autres contributions du recueil. Deux d'entre elles replacent l'œuvre de Virey dans les controverses qui animent la biologie de l'époque : Roselyne Rey montre comment le vitalisme de Virey peut se concilier avec sa philosophie dualiste, tandis que Goulven Laurent s'attache à saisir les éléments qui rapprochent son œuvre des conceptions transformistes, telles qu'elles se présentent au début du XIX ${ }^{\mathfrak{e}}$ siècle.

Les trois autres contributions étudient plus particulièrement l'anthropologie de Virey. Geneviève Fraisse traite sa représentation du partage des sexes; Claude Bénichou analyse sa conception de la psychologie et restitue par là un chapitre méconnu de l'histoire de cette discipline. Claude Blanckaert démonte les objectifs, la cohérence et les paradoxes de cette " histoire naturelle de l'homme ", dont le modèle est emprunté à Buffon. La tâche est rendue difficile par le caractère ouvertement raciste de certaines thèses de Virey. Claude Blanckaert rappelle les termes du débat qui opposait au $\mathrm{XIX}^{\mathrm{e}}$ siècle monogénistes et polygénistes, les uns affirmant, les autres niant l'unicité de l'espèce humaine. Virey se situe dans le camp polygéniste, puisqu'il considère que les races blanches et les races noires forment deux espèces différentes, mais il se rapproche sur beaucoup de points du monogénisme. C'est un franc-tireur inclassable que les anthropologues du milieu du siècle s'entendront à rejeter dans la préhistoire de leur discipline. Claude Blanckaert s'attache à toutes les subtilités de la pensée de Virey. Peut-être même lui prête-t-il un peu généreusement sa propre agilité dialectique et élude-t-il un peu vite des interprétations plus triviales, telles que le désir chez Virey de flatter les goûts supposés du public.

D'une façon générale, tandis que la philosophie de Virey est scrutée avec infiniment d'acuité, son travail de vulgarisateur, son rapport au public, ses procédés rhétoriques, son usage de l'illustration, restent dans l'ombre ou sont, au mieux, effleurées. Ceux qui voudront combler cette lacune, ou simplement prendre connaissance directement de l'œuvre de Virey trouveront dans la dernière partie une bibliographie d'une vingtaine de pages, qui ne prétend pas être exhaustive mais qui est cependant très fournie. La tâche était d'autant plus difficile et nécessaire qu'une grande partie des textes de Virey ici recensés sont des articles du Dictionnaire des sciences médicales (Paris, Panckoucke, 1812-1822) en 
60 volumes, ou du Nouveau dictionnaire d'histoire naturelle (Paris, Deterville, 1803 ) en 24 volumes. Cette bibliographie, la présence d'un index des noms propres, la qualité de la typographie et de la mise en page, concourent à faire de ce livre un ouvrage de référence sur un aspect méconnu de l'histoire des sciences.

Jean-Marc Drouin.

Francisco Javier Puerto Sarmiento, La Ilusión quebrada. Botánica sanidad y politica cientifica en la España ilustrada. Barcelone/Madrid, Serbal/CSIC, 1988. $19 \times 25,5,315$ p., bibliogr., index ("Libros del buen andar », 22).

Charles III, qui régna en Espagne de 1759 à 1788, fut un représentant de ce qu'on a appelé le "despotisme éclairé". Aidé de ses ministres Aranda et Floridablanca, il encourage l'agriculture, le commerce et expulse les Jésuites (1767). C'est dans ce contexte qu'il faut comprendre le projet de réforme des structures économiques, administratives et scientifiques de l'Espagne et de ses colonies dans lequel les sciences - et notamment la botanique - ont joué un rôle essentiel. M. Puerto Sarmiento, professeur d'histoire de la pharmacie à l'Université Complutense de Madrid, étudie la naissance de ce projet, son développement dans les colonies et son extension en Europe : il décrit le processus par lequel la botanique s'est institutionnalisée et celui qui a conduit les botanistes à s'affirmer en tant que véritables scientifiques. Au centre de cette volonté de changement se trouve le Real Jardín Botánico, dont Casimir Gómez Ortega fut le directeur de 1771 à 1801 . Gómez Ortega est le pivot de cette tentative dans laquelle réforme sanitaire, connaissance du monde naturel espagnol et américain, désir d'une meilleure utilisation de la flore exotique sont étroitement imbriqués. Pour mener à bien cette réforme, il a fallu améliorer la formation des botanistes, les ouvrir à l'Europe des Lumières, moderniser lẹs structures du système de santé et explorer la nature espagnole et américaine. Cette réforme fut presque une réussite puisque Humboldt, vers 1800 , s'émerveille de l'effort accompli par l'Espagne pour connaitre correctement le monde naturel. Mais, lorsqu'en 1801, Cavanilles prend la suite de Gómez Ortega, l'illusion, telle qu'on l'avait rêvee à l'époque de Charles III, se brise, car le Jardin botanique royal se consacre presque exclusivement à la science pure sans se soucier outre mesure de la politique scientifique concrète. Les botanistes continuent de travailler dans leur sphère purement scientifique et, parallèlement, les médecins, les chirurgiens, les pharmaciens, cantonnés dans leur domaine professionnel, se détachent du projet de reforme pour suivre des chemins particuliers.

Le livre de M. Puerto Sarmiento est très bien réalisé et présente toutes les qualités d'un livre scientifique digne de ce nom : clarté du propos, style efficace, bibliographie, index, appendices, notes abondantes. De plus, La Ilusión quebrada est un ouvrage fort bien illustré, quelquefois en pleine page couleur, ce qui en rend la lecture agréable, rapide et instructive. Quelques noms français sont mal orthographiés - d'Alambert pour d'Alembert (p. 163) ou Duhamel de Monceau 
pour Duhamel du Monceau (p. 167) - mais ce n'est qu'un détail dans un travail de haute tenue.

Hervé GUÉNOT.

Jean Rostand, Confidences d'un biologiste. Textes réunis et présentés par JeanLouis FisCher. Paris, La Découverte, 1987. 13,5 × 22, 245 p. (« Histoire des sciences ").

Par ces morceaux choisis, Jean-Louis Fischer a voulu montrer "l'étendue des connaissances ", " la variété des activités intellectuelles » et la pertinence toujours actuelle des réflexions de Jean Rostand. L'Avant-Propos de ce livre brosse d'abord un portrait rapide du savant, de sa carrière originale, de ses recherches expérimentales qui furent souvent en leur temps des recherches de pointe, et de son humanisme engagé. Les textes sont ensuite regroupés sous quatre rubriques : " Naissance d'une passion ", "L'Évolution de l'hérédité ", " Erreurs et vérités ", "Autoportrait d'un savant». On peut s'étonner de quelques choix : le texte sur "Zola, chercheur de vérité " placé en fin de la deuxième partie, ne semble guère avoir de rapports avec les trois autres, plus nettement didactiques; le texte "Science fausse et fausses sciences", qui retrace la vogue éphémère des rayons $\mathbf{N}$ et polémique contre le métapsychisme et le mitchourinisme, n'est sans doute pas un des meilleurs du "biologiste". Mais la sélection est dans l'ensemble heureuse : on a ici quelques beaux échantillons de la clarté des exposés de Rostand (II ${ }^{\mathrm{e}}$ partie surtout), de l'humour qu'il sait mettre dans ses plaidoyers aux enjeux les plus sérieux (voir texte $\mathrm{n}^{\circ} 2$ où un amusant discours "pro rana" devient un manifeste sur la patience et la passion de la recherche expérimentale), et de la manière dont il a su déployer ses méditations ou les ramasser en de précieuses formules (voir surtout III $^{\mathrm{e}}$ partie).

Le titre choisi pour ce recueil - Confidences... - ne doit point en masquer la portée. Par-delà le lyrisme du discours personnel où l'humour et l'humeur sont habilement maniés, ces textes construisent en fait un véritable discours de la méthode où s'imbriquent réflexions épistémologiques et déontologiques. On retiendra, du premier texte par exemple, que le récit des émerveillements de l'enfance débouche sur un plaidoyer pour " l'éminente dignité de la vulgarisation ": Rostand a une conception exigeante de ce qu'il appelle "la science diffusée " et ce livre tout entier l'illustre. On retiendra aussi le texte intitulé « De la recherche en biologie " qui est en fait une réflexion approfondie, exemples à l'appui, sur les démarches heuristiques, leur diversité, leur complexité, les parts du hasard et des méthodes, le rôle des facteurs matériels ou/et celui des facteurs caractériels du chercheur, etc. On retiendra aussi, épars dans bien des textes, les multiples plaidoyers de Rostand contre les frontières disciplinaires : il réclame le rapprochement des sciences et des lettres, et, côté scientifique, il veut un enseignement qui encourage l'observation et ne sélectionne point sur les seules aptitudes mathématiques. De tous ces textes, on retiendra encore et surtout que si Rostand 
défend âprement la passion de savoir - les longues patiences du "chercheur " besognant et bricolant de façon plus ou moins programmée et les " grisantes " "jouissances " du «trouveur » - , il sait éviter d'entonner naïvement l'hymne triomphaliste sur l'objectivité, la neutralité et les progrès obligés de la science : point question de masquer les difficultés des exigences laborieuses de la recherche, les pièges des expérimentations sollicitées, ou ceux des théorisations hâtives simplifiantes ou préjugées. Il est clair, enfin, que par-delà ses recherches sur les petits animaux, Rostand a une conscience aiguë des problèmes multiples dans lesquels se débat "l'animal humain ". Soit, par exemple, ces deux aphorismes: " Le règne de la science a ouvert une sorte d'époque glaciaire dans l'histoire spirituelle de notre espèce : il n'est pas sûr que la frileuse âme humaine puisse résister au climat rigoureux de la raison "... « J'aime qu'on suffoque dans la raison mais qu'on s'y tienne ". Ce chaud/froid métaphysique montre que les confidences de ce «biologiste ", qui n'oublie pas d'être homme, offrent à penser à tous. Il est de sereines « inquiétudes » qui valent d'être partagées.

Annie PETrT.

Théories biologiques, éthique et expérimentation en médecine. Dir. Hervé BARREAU. Paris, Ed. du C.N.R.S., 1988. $16 \times 24,264$ p. (« Fondements des sciences ").

Ce recueil contient un ensemble d'articles extrêmement divers. La synthèse générale, qu'en propose Hervé Barreau dans son introduction intitulée « Le savoir de la vie " (p. 9-21), le conduit à poser le problème d'une articulation entre éthique de la vie et pouvoir scientifique. Si la vie est source de connaissance, la connaissance est-elle source de vie? Il semble donc qu'il faudrait amorcer une réflexion éthique générale qui ne s'en tiendrait pas aux questions de l'application ou de l'expérimentation du savoir, mais qui interrogerait aussi, en amont, la recherche dans le moment même de la construction des théories. On regrettera alors que la diversité des textes proposés ici ne recèle pas les éléments d'une telle synthèse entre problèmes théoriques et éthiques en biologie. Cinq articles sur des questions essentiellement théoriques sont suivis de deux articles qui ne traitent que des problèmes éthiques de l'expérimentation sur l'homme et l'animal.

Dans " Faits biologiques en quête de théories" (p. 25-79), Charles Marx se risque à un panorama général de l'histoire de la biologie pour montrer la nécessité d'une approche systémique de l'organisation du vivant. Après avoir présenté les quelques premiers résultats d'une théorie générale des systèmes, il élargit encore la question en remarquant que le besoin de comprendre, c'est-à-dire de donner un sens aux phénomènes, et la tendance à penser le vivant en termes finalistes, sont inhérents à l'homme. Si l'on reconnaît pleinement la nature biologique de l'être humain, il est impossible d'esquiver le problème de la formation de projets dans les systèmes biologiques. Charles Marx conclut en se demandant s'il n'y aurait pas une impossibilité radicale pour l'homme, en tant qu'être vivant, à 
comprendre de tels "projets biologiques". Une impossibilité qui découlerait du paradoxe logique d'une partie de la nature qui prétendrait saisir le tout qui la contient.

Dans "Symétrie et morphogenèse " (p. 81-110), Yves Bouligand nous fait découvrir une étonnante diversité d'états intermédiaires entre le solide et le liquide, entre l'ordre et le désordre. Il y ajoute une bonne bibliographie historique de la question un peu oubliée des symétries en biologie.

Dans son article sur " La Phyllotaxie et le rêve du cristal vivant " (p. 111-154), Hervé Le Guyader donne un intéressant historique de la phyllotaxie, c'est-à-dire l'étude des arrangements des feuilles sur une tige. Cette question fut l'occasion de la première introduction des mathématiques et de la géométrie dans l'étude des êtres vivants. Ces recherches se prolongent à l'heure actuelle par la simulation sur ordinateur de "systèmes générateurs de cartes à réécriture parallèle ", et par l'emploi de la théorie des automates.

Jean-Paul Le Pennec donne un rapide résumé des derniers progrès et problèmes de la biologie moléculaire dans «Quelques données récentes en génétique moléculaire " (p. 155-169).

Enfin, dans "Le concept d'auto-organisation en biologie théorique » (p. 171195), Henri Atlan montre comment l'observation du comportement collectif organisé d'un réseau d'automates booléens arrangés au hasard permet de dépasser la simple notion probabiliste d'information (au sens de Shannon), en suggérant comment elle pourrait acquérir une signification. En effet, de tels réseaux semblent fonctionner comme des reconnaisseurs de certaines classes de séquences de signaux.

Les deux communications suivantes sont d'ordre éthique. Elles ne concernent pas les problèmes d'actualité comme les "manipulations génétiques" ou les fécondations in vitro, mais simplement l'expérimentation sur l'homme et l'animal.

Le plaidoyer d'Alexandre Petrovic dans la « Recherche sur l'animal et médecine pour l'homme " (p. 199-240), bien qu'il manque certainement d'un approfondissement philosophique, est une vigoureuse défense de l'expérimentation sur l'animal.

Le dernier article est écrit par Anne Fagot-Largeault dont on connaît les exposés lumineux sur l'histoire et les développements de l'analyse bayésienne. Dans ce texte, "La décision médicale : recherche et pratique " (p. 241-259), elle rappelle comment les développements de la recherche en Intelligence Artificielle ont entraîné tout un travail d'éclaircissement sur les techniques de prise de décision, en particulier dans les actes médicaux. Mais quand le médecin doit aussi se faire expérimentateur, la mesure de l'avantage de chaque décision se trouve singulièrement compliquée par la nécessité d'évaluer le "coût éthique " du savoir relativement à celui de la santé du malade. Anne Fagot-Largeault présente alors les diverses problématiques éthiques sous-jacentes au choix d'une des différentes logiques de décision possibles : catégorique, statistique, probabiliste (bayésienne), ou les méthodes de « logique floue». 
Isabelle Wohnlich-Despaigne, Les Historiens français de la médecine au $X I X^{e}$ siècle et leur bibliographie. Préf. de Pierre Chaunu. Paris, Vrin, 1987. $16 \times 24,423$ p. (« L'histoire des sciences; textes et études »).

$\mathbf{M}^{\text {me }}$ Wohnlich a fait œuvre utile. La partie la plus importante de son ouvrage est constituée par un index de 7571 références de travaux d'histoire de la médecine, effectués par des historiens du XIX ${ }^{\mathrm{e}}$ siècle. Ces derniers sont classés par ordre alphabétique et numéral. D'autres index complètent cette bibliographie d'auteurs français, à savoir des matières, des lieux et des personnes cités ou étudiés. Comme toute bibliographie volumineuse, fût-elle très minutieuse, celle-ci est incomplète et certaines provinces comme la Corse n'ont pas trouvé une grande place ici. Mais à peine ai-je écrit cette discrète critique que je me dois aussitôt de la tempérer. Ce travail est important, remarquable et doit être apprécié. Pierre Huard a d'ailleurs guidé les premiers pas de cette œuvre mais malheureusement n'a pu en voir l'aboutissement.

L'autre intérêt de ce livre réside dans son introduction. L'auteur rappelle, en effet, qu'à partir de $1685,90 \%$ des ouvrages sont rédigés en français, souligne que la renaissance médicale n'a commencé qu'au $x^{\prime}{ }^{e}$ siècle pour se terminer en 1816 et insiste justement sur le rôle essentiel de Magendie. La chaire d'histoire de la médecine ne trouve guère de grâce aux yeux d'Isabelle Wohnlich du fait de son rôle accessoire, sauf durant la courte période où Daremberg en fut le titulaire. La classification des historiens proposée en praticiens, savants, érudits, philologues et journalistes est très intéressante car très didactique et l'auteur n'ignore pas qu'elle est cependant par trop réductrice car bien des écrivains, des enseignants, des chercheurs échappent à ces classifications. On lira de même avec intérêt et profit les pages consacrées à la méthodologie et à l'histoire de la bibliographie depuis 1645, date de l'édition de la Bibliotheca Universalis de Conrad Gesner.

Un livre important; une référence.

Vincent-Pierre CoMrrI.

" L'homme et les virus », Impact. Science et société, 150, 1988.

La revue internationale Impact, publiée par l'U.N.E.S.C.O., a pour mission de montrer les différentes façons dont l'évolution des sciences et des techniques influe sur la vie des individus et des sociétés. Rien de surprenant donc qu'un numéro spécial soit consacré à la lutte de l'homme contre les virus, les seuls êtres biologiques qu'il n'a pas réussi à dominer. Au moment même où l'homme proclame sa victoire définitive sur l'ancien virus de la variole, tueur historique particulièrement efficace, un virus jusqu'alors inconnu déclenche une offensive épidémiologique à nulle autre pareille. Les ravages du virus de l'immunodéficience acquise ont surpris aussi bien les savants que le commun des mortels : ils ont cru que la biotechnologie moderne les avait mis à l'abri des catastrophes de 
ce genre. Épidémie à la fois terrible et exemplaire, le sida est devenu aussi un symbole et un paradigme. Il justifie l'intérêt de toutes les collectivités humaines pour ce monde d'êtres invisibles qui se situent à la limite du vivant. Parasites cellulaires absolus, dépourvus de la plupart des fonctions physiologiques et ne manifestant leur "vie » que par le truchement des appareils cellulaires piratés, les virus agissent au niveau suprême de l' " information biologique ».

Les virus suscitent d'abord un intérêt d'ordre pratique : ils sont aujourd'hui l'une des principales causes de maladie et de décès. Ils méritent d'être connus aussi pour des raisons d'ordre théorique. Dans la lutte actuelle entre l'homme et les virus, l'intelligence, c'est-à-dire le traitement de l'information stockee dans les réseaux des neurones, s'oppose aux ruses qui dérivent du traitement de l'information contenue dans les génomes.

Des spécialistes éminents traitent dans ce volume des aspects les plus saisissants de la virologie : Hugues de Thé décrit les principales formes virales et donne une explication globale de leurs stratégies de réplication et de piratage des cellules humaines, animales ou végétales. Oyewale Tomori, médecin africain rompu au travail dans les régions tropicales, trace un tableau épidémiologique et clinique remarquable de la fièvre de Lassa et d'autres affections virales hémorragiques. Pierre Sonigo, spécialiste de la biologie moléculaire, parle avec clarté et compétence du virus de l'hépatite $\mathrm{B}$; son propos illustre le triomphe du génie génétique qui a permis, d'un côté, de rapides progrès dans la compréhension du cycle viral et, de l'autre, la production d'un vaccin efficace. Un virologiste chinois très renommé, Zhu Ji-ming, décrit les viroses qui sévissent dans son pays. Frank Fenner, l'un des principaux responsables de la campagne mondiale d'éradication de la variole, dit dans un texte clair et dense pourquoi et comment l'on a réussi à vaincre cette maladie. La contribution de Jonathan Mann sur le sida illustre les fondements idéologiques du programme international de la lutte contre cette maladie. Enfin, quelques articles sont consacrés aux maladies virales des poulets, des bovins, des petits ruminants et des végétaux.

Dans son ensemble, ce fascicule offre une bonne mise au point des problèmes médicaux et sociaux de la virologie contemporaine. Les textes sont pondérés, d'une précision remarquable et d'une haute qualité scientifique, déblayés autant que cela se peut du jargon professionnel. Toutefois, les lecteurs ayant une prédilection pour des problèmes biologiques généraux, " philosophiques ", resteront sur leur faim. Il y aurait tellement de choses encore à dire sur le rôle des virus dans l'évolution des êtres vivants, sur leurs stratégies de survie, sur l'importance de leur étude pour la compréhension de l'hérédité et de la régulation cellulaire et même sur la définition générale de la vie, largement tributaire aujourd'hui des acquis récents de la virologie!

Mirko D. GRMEK. 
Mirko D. GrmeK, Histoire du sida. Début et origine d'une pandémie actuelle. Paris, Payot, 1989. $14 \times$ 22,5, 392 p., notes, document, bibliogr. (« Médecine et sociétés Payot ").

L'ouvrage de Mirko Grmek consacré à l'histoire du sida est exemplaire à plus d'un titre : exemplaire de ce que peut produire, aujourd'hui, un historien de la médecine sur un objet dont l'actualité est brûlante ; exemplaire, par ses qualités, de ce que peut être une analyse du processus par lequel la médecine construit une entité pathologique tout à la fois nouvelle (au sens d'inconnue jusque-là) et profondément originale par ses mécanismes d'action; exemplaire, par ses défauts, des pièges qui guettent celui qui s'aventure sur le terrain de l'explication à prétention globalisante de la survenue d'une épidémie.

M. Grmek, et d'autres avec lui, a mis en relief à propos du sida, l'extrême efficacité dont a su faire preuve le monde médico-scientifique dans l'individualisation de la nouvelle maladie et la découverte de l'agent infectieux, grâce à l'articulation d'approches biomédicales, dont la complémentarité avec les travaux épidémiologiques et cliniques s'est avérée remarquable. Il me semble que le travail qu'il a accompli n'est pas sans présenter d'analogie avec ce phénomène. Le « tour de force " consistant à produire si rapidement une histoire médicale du sida témoigne, à sa façon, de l'efficacité dont est capable le monde des historiens de la médecine, grâce en partie aux conditions de plus en plus performantes d'accessibilité aux documents (plus de 900 articles cités dont la plupart sur la période 1982-1987!).

La première moitié du livre est proprement passionnante. Par un effet de construction que ne désavoueraient pas les meilleurs écrivains de romans policiers, l'auteur nous fait suivre pas à pas la course poursuite à laquelle se livrent le «fléau » et ses pisteurs. Il décrit avec talent la progression de l'épidémie avec ses temps forts et ses moments de latence, et la mise en place progressive des pièces d'un puzzle, qui malgré les hésitations, les fausses routes, les aveuglements, finira par dévoiler les figures de la maladie, ses modes de transmission, ses mécanismes d'action et son agent causal.

Dans la seconde partie, l'ambition qui s'affiche est d'une autre ampleur. S'engageant dans la recherche des signes susceptibles de témoigner d'éventuelles relations entre le genre humain et le virus HIV, antérieures à l'épidémie actuelle, relations que la médecine, faute de posséder les outils conceptuels adéquats aujourd'hui à sa disposition, n'avait pu repérer, $M$. Grmek substitue à la pauvreté des données la force argumentaire de spéculations théoriques. Il utilise un modèle d'explication construit autour d'un concept qui lui est propre, celui de pathocenose, sans enrichir ni la compréhension de l'apparition de l'infection HIV, ni son modèle.

S'il n'y a pas lieu ici d'entrer dans l'examen critique des nombreuses assertions que véhiculent ce concept de pathocenose et son postulat d'un état d'équilibre entre l'espèce humaine et les divers agents infectieux menaçant son existence, il n'en demeure pas moins qu'on peut légitimement reprocher à l'auteur d'avoir, en l'appliquant à l'histoire du sida, fait la part trop belle aux idées reçues et aux lieux communs idéologiques. Les références hâtives (et, pour l'essentiel, médicales) aux 
excès de la "révolution sexuelle" des vingt dernières années, la vision non exempte de préjugés moralisateurs (cf. l'usage non contrôlé du terme de promiscuité) d'un monde homosexuel autrefois contraint à la modération de ses appétits par la répression, s'adonnant sans retenue à un stakanovisme sexuel, l'invocation d'un changement des mœurs ancestrales (sic) des Africains, tiennent lieu d'analyse historico-sociologique. Comme si, en quittant son terrain, la médecine, pour embrasser l'analyse du monde social, l'historien oubliait la rigueur et les contraintes de l'analyse scientifique.

Patrice PINELL. 